\title{
THE IDENTITY OF WEAK AND STRONG EXTENSIONS OF PSEUDO-DIFFERENTIAL OPERATORS ${ }^{1}$
}

\author{
FERNANDO CARDOSO
}

ABstract. In this paper we consider first order Kohn and Nirenberg pseudo-differential operators, define their weak and strong $L_{2}$ extensions and prove, by standard mollifiers and Fourier transform techniques, the identity between them.

In this article we shall consider operators of the form

$$
L=\sum_{1}^{n} A_{j} D_{j}
$$

where $A_{j}=a_{j}(x ; D)$ is a pseudo-differential operator of order zero and $D_{j}=-(-1)^{1 / 2} \partial / \partial x^{j}$.

We denote by $S$ the space of $C^{\infty}$ complex (scalar, vector or matrix) valued functions $u(x), x=\left(x^{1}, \cdots, x^{n}\right)$, defined in $R^{n}$ which together with all their derivatives die down faster than any power of $|x|$ at infinity.

$A_{j}$ maps $S$ continuously into $S$ and is defined by

$$
A_{j} u(x)=\int e^{i x \cdot \xi} a_{j}(x ; \xi) \mathfrak{a}(\xi) d \xi,
$$

where

$$
\hat{u}(\xi)=\frac{1}{2 \pi} \int e^{-i x \cdot \xi} u(x) d x
$$

is the Fourier transform of $u(x)$. Here $u(x)=\left\{u_{1}, \cdots, u_{k}\right\}$ and as usual $\xi=\left(\xi_{1}, \cdots, \xi_{n}\right),(x \cdot \xi)=\sum x^{j} \xi_{j}$ and $d x=d x^{1} \cdots d x^{n}, d \xi$ $=d \xi_{1} \cdots d \xi_{n}$. We make also use of the familiar notation $D=\left(D_{1}, \cdots, D_{n}\right), D^{\mu}=D_{1}^{\mu} \cdots D_{n^{n}}^{\mu}$ for $\mu=\left(\mu_{1}, \cdots, \mu_{n}\right)$ a multiindex with the $\mu_{j}$ integers $\geqq 0$. We assume that the symbol $a_{j}(x ; \xi)$

Received by the editors August 13, 1970.

AMS 1969 subject classifications. Primary 3523.

Key words and phrases. Pseudo-differential operator, weak and strong extensions, mollifiers, Fourier transforms.

1 These results are contained in the author's doctoral dissertation written under the guidance of Professor K. O. Friedrichs at the Courant Institute of Mathematical Sciences, New York University. The author was supported by the National Research Council of Brazil. 
of $A_{j}$ is a $C^{\infty} k \times k$ matrix, homogeneous of degree zero in $\xi$ for $|\xi| \geqq 1$ and independent of $x$ for $|x|$ large:

$$
a_{j}(x ; \xi)=a_{j}(\infty ; \xi)+a_{j}^{0}(x ; \xi)
$$

where $a_{j}^{0}(x ; \xi)$ has bounded $x$ support and as a function of $x$ belongs to $S$ uniformly in $\xi$, i.e. for any integer $p$,

$$
\left|D^{\mu} a_{j}^{0}(x ; \xi)\right| \leqq \frac{\text { const }}{\left(1+|x|^{2}\right)^{p}} .
$$

In fact, in [2] it is also assumed that the same is true for all derivatives in $\xi$.

A formal adjoint $L^{(*)}$ of $L$ can be formed with the aid of the "local" adjoint $L^{(*)}=\sum_{1}^{n} D_{j} A_{j}^{(*)}$ defined on $\mathcal{S}$ so that $\left(L^{(*)} v, u\right)=(v, L u)$ holds for all $v$ and $u$ in $\delta ;($,$) is the inner product in L_{2}\left(R^{n}\right)$, the corresponding norm being denoted by \|\| . As remarked in [2], $A_{j}^{(*)}$ is the "reversed operator" $A_{j}^{\text {TCR }}$ given by

$$
\left(A_{j}^{\mathrm{TCR}} v\right)^{\wedge}(\xi)=\int e^{-i x \cdot \xi} a_{j}^{\mathrm{TC}}(x ; \xi) v(x) d x
$$

where $a_{j}^{\mathrm{TC}}(x ; \xi)$ is the conjugate transpose of $a_{j}(x ; \xi)$. We shall introduce the notions of weak and strong extensions of $L$ (weak and strong solutions of $L u=f$ ) which will be denoted throughout this paper by $\tilde{L}$ and $\bar{L}$, respectively.

Definition 1. Let $f$ be a square integrable function, i.e. $f \in L_{2}\left(R^{n}\right)$; the square integrable function $\tilde{u}$ is said to be a weak solution of $L u=f$ if $\left(L^{(*)} v, \tilde{u}\right)=(v, f)$ holds for all $v$ in $s$.

Definition 2. Let $\bar{u}$ and $f$ be square integrable functions; $\bar{u}$ is said to be a strong solution of $L u=f$ if there exists a sequence $\left(u_{j}\right)$ in $\delta$ such that

$$
\left\|u_{j}-\bar{u}\right\|+\left\|L u_{j}-f\right\| \rightarrow 0 \text { as } j \rightarrow \infty .
$$

That a strong solution of $L u=f$ is also a weak one follows easily from the definitions.

To prove the converse we need two lemmas but first we introduce a class of smoothing operators called "mollifiers." Choose $j(x)$ in $\mathcal{S}$ such that its Fourier transform

$$
\begin{aligned}
\hat{\jmath}(\xi)=1 & \text { if }|\xi| \leqq 1, \\
& =0 \text { if }|\xi| \geqq 2 .
\end{aligned}
$$

Define $j_{\epsilon}(x)$ in such a way that 


$$
\hat{\jmath}_{\epsilon}(\xi)=\hat{\jmath}(\epsilon \xi) .
$$

The mollifier $J_{\epsilon}$ is then defined as convolution with $j_{\epsilon}$ :

$$
J_{\epsilon} u=j_{\epsilon} * u=\int j_{\epsilon}(z) u(x-z) d z .
$$

LEMma 1. The commutator $\left[J_{\epsilon}, L\right]$ is an operator of order zero, i.e. it maps $L_{2}\left(R^{n}\right)$ continuously into $L_{2}\left(R^{n}\right)$. Also $\|\left[J_{\epsilon}, L\right][\| \leqq C$, where $C$ is a constant independent of $\epsilon$.

Proof. We can write

$$
\left[J_{\epsilon}, L\right]=\sum_{1}^{n}\left[J_{\epsilon}, A_{j} D_{j}\right]=\sum_{1}^{n}\left[J_{\epsilon}, A_{j}\right] D_{j}
$$

therefore we need just prove the lemma for $\left[J_{\epsilon}, A_{j}\right] D_{j}$. For any $v \in \mathcal{S}$,

$$
\left[J_{\epsilon}, A_{j}\right] D_{j} v=J_{\epsilon} A_{j} D_{j} v-A_{j} J_{\epsilon} D_{j} v=j_{\epsilon} * A_{j} D_{j} v-A_{j}\left(j_{\epsilon} * D_{j} v\right) .
$$

From this it follows by taking Fourier transforms

$$
\begin{aligned}
\left(\left[J_{\epsilon}, A_{j}\right] D_{j} v\right)^{\wedge}(\xi) & =\hat{\jmath}(\xi)\left(A_{j} D_{j} v\right)^{\wedge}(\xi)-\left(A_{j}\left(j_{\epsilon} * D_{j} v\right)\right)^{\wedge}(\xi) \\
& =\int\left(a_{j}^{0}\right)(\hat{\xi}-\eta ; \eta) \eta_{j}\left(\hat{\jmath}_{\epsilon}(\xi)-\hat{\jmath}_{\epsilon}(\eta)\right) \hat{v}(\eta) d \eta=g_{\epsilon}(\xi) .
\end{aligned}
$$

We have to estimate the $L_{2}$-norm of $g_{\epsilon}(\xi)$ in terms of the $L_{2}$-norm of $\hat{v}(\eta)$. By the Holmgren theorem, it suffices to show that the integrals with respect to $\xi$ and $\eta$ of the kernel

$$
\left|\left(a_{j}^{0}\right)^{\wedge}(\xi-\eta ; \eta)\right|\left|\eta_{j}\right|\left|\hat{\jmath}_{\epsilon}(\xi)-\hat{\jmath}_{\epsilon}(\eta)\right|
$$

are bounded by constants independent of $\eta$ and $\xi$. Because of the fact that $a_{j}^{0}(x ; \xi) \in \mathcal{S}$ uniformly in $\xi$, we observe first that for any positive integer $p$

$$
\left|\left(a_{j}^{0}\right)^{\wedge}(\xi-\eta ; \eta)\right| \leqq \frac{\text { const }}{\left(1+|\xi-\eta|^{2}\right)^{p+1}} .
$$

On the other hand,

$$
\left(\hat{\jmath}_{\epsilon}(\xi)-\hat{\jmath}_{\epsilon}(\eta)\right) \eta=\xi \hat{\jmath}_{\epsilon}(\xi)-\eta \hat{\jmath}_{\epsilon}(\eta)-(\xi-\eta) \hat{\jmath}_{\epsilon}(\xi) .
$$

Since $\xi \cdot(d / d \xi) \hat{\jmath}_{\epsilon}(\xi)$ is bounded by a constant independent of $\xi$ so is

$$
\frac{d}{d \xi} \cdot \xi \hat{\jmath}_{\epsilon}(\xi)=\hat{\jmath}_{\epsilon}(\xi)+\xi \cdot \frac{d}{d \xi} \hat{\jmath}_{\epsilon}(\xi)
$$

and from the mean-value theorem we obtain 


$$
\begin{aligned}
\left|\left(\hat{\jmath}_{\epsilon}(\xi)-\hat{\jmath}_{\epsilon}(\eta)\right) \eta_{j}\right| & \leqq\left|\left(\hat{\jmath}_{\epsilon}(\xi)-\hat{\jmath}_{\epsilon}(\eta)\right) \eta\right| \leqq \text { const }|\xi-\eta| \\
& \leqq \text { const }\left(1+|\xi-\eta|^{2}\right) .
\end{aligned}
$$

Therefore with $p$ large, we establish immediately the desired estimate for the integral of (3) with respect to either $\xi$ or $\eta$. Since these integrals are clearly bounded by constants independent also of $\epsilon$,

$$
\left\|\left[J_{\epsilon}, L\right] v\right\| \leqq C\|v\|
$$

holds for all $v \in \mathcal{S}$, where $C$ is a constant independent of $\epsilon$. Because $\mathcal{S}$ is dense in $L_{2}\left(R^{n}\right)$, we finally have

$$
\left\|\left[J_{\epsilon}, L\right] u\right\| \leqq C\|u\|
$$

for all $u \in L_{2}\left(R^{n}\right)$. This completes the proof of Lemma 1.

Lemma 2. For every $u$ in the domain of $\tilde{L}$

$$
\left\|\left[J_{\epsilon}, \tilde{L}\right] u\right\| \rightarrow 0 \text { as } \epsilon \rightarrow 0 .
$$

Proof. Suppose $v \in C_{0}^{\infty}\left(R^{n}\right)$ (the space of $C^{\infty}$ functions which have compact support). We may write

$$
\begin{aligned}
\left|\left(\left[J_{\epsilon}, A_{j} D_{j}\right] v\right)^{\wedge}(\xi)\right|=\left|g_{\epsilon}(\xi)\right| \\
=\left|\int\left(a_{\jmath}^{0}\right)^{\wedge}(\xi-\eta ; \eta)\left(\hat{\jmath}_{\epsilon}(\xi)-\hat{\jmath}_{\epsilon}(\eta)\right) \eta_{j} \hat{v}(\eta) d \eta\right| \\
\quad \leqq \text { const } \epsilon \int\left|(\xi-\eta)\left(a_{j}^{0}\right)^{\wedge}(\xi-\eta ; \eta)\right|\left|\left(D_{j} v\right)^{\wedge}(\eta)\right| d \eta
\end{aligned}
$$

since $\left|\hat{\jmath}_{\epsilon}(\xi)-\hat{\jmath}_{\epsilon}(\eta)\right|=|\hat{\jmath}(\epsilon \xi)-\hat{\jmath}(\epsilon \eta)| \leqq$ const $\epsilon|\xi-\eta|$. But

$$
\left|(\xi-\eta)\left(a_{j}^{0}\right)(\hat{\xi}-\eta ; \eta)\right| \leqq C_{p}\left(1+|\xi-\eta|^{2}\right)^{->}
$$

for any $p$, uniformly in $\eta$. Thus, since $v \in C_{0}^{\infty}\left(R^{n}\right)$,

$$
\left\|g_{\epsilon}\right\| \leqq \text { const } \epsilon\left\|D_{j} v\right\|
$$

which implies that

$$
\left\|g_{\epsilon}\right\| \rightarrow 0 \quad \text { as } \epsilon \rightarrow 0 .
$$

By Parseval's relation, also,

$$
\left\|\left[J_{\epsilon}, A_{j} D_{j}\right] v\right\| 0 \quad \text { as } \epsilon \rightarrow 0
$$

and consequently

$$
\left\|\left[J_{\epsilon}, L\right] v\right\| \rightarrow 0 \quad \text { as } \epsilon \rightarrow 0 .
$$

Using Lemma 1 and the fact that $C_{0}^{\infty}\left(R^{n}\right)$ is dense in $L_{2}\left(R^{n}\right)$, we easily 
verify that $\left\|\left[J_{c}, \tilde{L}\right] u\right\| \rightarrow 0$ as $\epsilon \rightarrow 0$ for all $u$ in the domain of $\tilde{L}$. Q.E.D.

From Lemma 2 and the fact that [1]

$$
\left\|J_{\epsilon} u-u\right\| \rightarrow 0 \quad \text { as } \epsilon \rightarrow 0
$$

for all $u \in L_{2}\left(R^{n}\right)$ we finally conclude that

TheOREM 1. $\tilde{L}=\bar{L}$.

Proof. Assume that $\tilde{L} \tilde{u}=f$, that is $\tilde{u}$ is a weak solution of $L u=f$. Set $u_{j}=J_{\epsilon_{j}} \tilde{u}, \epsilon_{j} \rightarrow 0$ as $j \rightarrow \infty$. By (5),

$$
\left\|u_{j}-\tilde{u}\right\| \rightarrow 0 \text { as } j \rightarrow \infty
$$

and since $\left\|L u_{j}-f\right\|=\left\|L J_{\epsilon_{j}} \tilde{u}-f\right\| \leqq\left\|L J_{\epsilon_{j}} \tilde{u}-J_{\epsilon_{j}} \tilde{L} \tilde{u}\right\|+\left\|J_{\epsilon_{j}} f-f\right\| \rightarrow 0$ as $j \rightarrow \infty, \tilde{u}$ is also a strong solution.

REMARK. One readily verifies that the special form (1) of the operator $L$ plays no role in the proof of Lemmas 1 and 2 . We might as well have considered an operator $L$ of the form $L=A+B$ where $A$ and $B$ are pseudo-differential operators of order one and zero, respectively, applicable to vector valued functions $u=\left(u_{1}, \cdots, u_{k}\right)$ defined in some Euclidean space.

\section{BIBLIOGRAPHY}

1. K. O. Friedrichs, The identity of weak and strong extensions of differential operators, Trans. Amer. Math. Soc. 55 (1944), 132-151. MR 5, 188.

2. J. J. Kohn and L. Nirenberg, An algebra of pseudo-differential operators, Comm. Pure Appl. Math. 18 (1965), 269-305. MR 31 \#636.

Universidade Federal de Pernambuco, Recife, Brazil 